\title{
Professional Training of Intellectual Disabled Person by Holographic Image of Competent Healthy Specialist
}

\author{
Evgeniy Bryndin \\ Research Centre "NATURAL INFORMATIC", Technological Platform Future Medicine, Novosibirsk, Russia \\ Email address: \\ bryndin15@yandex.ru \\ To cite this article: \\ Evgeniy Bryndin. Professional Training of Intellectual Disabled Person by Holographic Image of Competent Healthy Specialist. \\ International Journal of Psychological and Brain Sciences. Vol. 6, No. 3, 2021, pp. 44-51. doi: 10.11648/j.ijpbs.20210603.12
}

Received: July 5, 2021; Accepted: July 14, 2021; Published: July 21, 2021

\begin{abstract}
The article is devoted to professional training of intellectual disabled person for competent performance of duties in the industrial and social sphere. The purpose of the article is to show how you can make a professional human assistant from an intellectual disabled person. An intellectual disabled person is a person who has limited opportunities for his personal life in society due to his intellectual, mental and mental disabilities. An intellectual disabled person can be trained in professional activities using the holographic image of a healthy person specialist. Professional training of an intellectual disabled person is carried out by tuning it in a holographic professional way by loading through a sensor system using magnetic resonance and holographic technologies and resonators. The hologram of a professional specialist becomes a professional activity program for an intellectual disabled person. It forms a projection of the information activity process of a competent competent healthy specialist. The intellectual disabled person can form and tune semantic memory with professional knowledge also through the sensory system using magnetic resonance and holographic technologies and resonators. A trained intellectual disabled person becomes a professional healthy performer. At the same time, it forms its own time in accordance with the centers processing information, and their entire set, which constitutes a single whole in the holographic projection. Thanks to his processing centers, he forms his own environment from the inside. Activation of a trained healthy performer for professional activity is carried out by a specialist through a mental neurointerface. Further communication between them in the process of production activities takes place through mental neurointerfaces.
\end{abstract}

Keywords: Intellectual Disabled, Holographic Image, Vocational Training, Magnetic Resonance Technologies

\section{Introduction}

Solving the problem of professional rehabilitation of an intellectual disabled person while limiting his sociopsychological abilities falls into the category of complex problems. First of all, because the object of influence is a person with mental and psychophysiological features, and, secondly, the change, or compensation of these characteristics, is realized by complex systems, which, in turn, include internal structures, functions and processes. One of the solutions is a method of comprehensive holographic professional rehabilitation of an intellectual disabled person, industrial and social work with this category of the population.

Integrated rehabilitation is a systemic concept that has scientific foundations and methods of practical implementation, which includes interconnected elements of the conceptual plan and communication technologies of production and social work in solving specific issues.

Currently, the development of effective technologies, methods and forms of socialization of a person with disabilities at all stages of his development is of great importance. The effective implementation of such social technologies should be based on the concept of an active and creative model of the individual as the main condition for successful rehabilitation, successful social work with an intellectual disabled person.

In the process of comprehensive rehabilitation, specialists of various levels participate - from organizers and managers to specific performers: doctors, rehabilitologists, social workers, rehabilitation specialists, teachers, psychologists and many other specialists. The concept of rehabilitation includes both prevention, treatment, adaptation to life and work after illness, and a personal approach to a sick person. 
Comprehensive rehabilitation of persons with disabilities is a complex multi-component system in which medical, social, psychological and professional aspects are equivalent. The rehabilitation system as a whole includes multiple structures that implement processes that aim to provide persons with disabilities with the opportunity to achieve the optimal physical, intellectual, social and mental level of activity, to support and thereby change lives and expand the scope of their independence. Thus, the process of integrated rehabilitation involves a wide range of measures and activities both activating a person with a disability and changing society in order to create conditions for persons with disabilities.

While setting the task of integrating citizens with disabilities into social society through an effective rehabilitation process, it should be borne in mind that this goal will be achieved through the effective implementation of various components of rehabilitation: medical, professional, psychological, sociocultural. The full integration of intellectual persons with disabilities into professional and socio-cultural life requires the use of high technology. In many cases, there are no technical, medical, social and other means that compensate for the complete defects: deafness, blindness, mental retardation, musculoskeletal disorders). Therefore, the specificity of social ties and relationships of persons with disabilities in society remains, and the processes of segregation and integration of persons with disabilities will be solved by the effectiveness of social and productive work with intellectual persons with high technologies.

An intellectual disabled person is a person who has limited opportunities for his personal life in society due to his intellectual, mental and mental disabilities.

In general, the following main areas of comprehensive rehabilitation of persons with disabilities can be identified:

1. Educational, the purpose of which is to eliminate the shortcomings of the existing attitude of society towards people with disabilities and people with disabilities towards society, a change in morals, politics, life, mentality in this area of interpersonal and social relations.

2. Leisure activities, the purpose of which is to organize and provide leisure activities to meet the spiritual and physical needs of persons with disabilities by providing meaningful time for persons with disabilities and their families.

3. Corrective, the purpose of which is to completely or partially eliminate or compensate the restrictions on the life and development of the person due to health reasons, as well as due to pedagogical and educational omissions. At the same time, it should be noted:

a) Corrective and educational impact, which includes the inculcation of social norms, by which social rules and sustainable forms of social interaction of people at the level of macro- and microcollections are meant;

b) Corrective and developmental impact focused on the comprehensive, holistic development of the individual, which implies the active development of cultural riches and the self-development of the creative personality;

c) corrective-educational, which is focused on increasing the education of the individual.

4. Cognitive impact focused on the acquisition by persons with disabilities of knowledge, concepts about real phenomena, awareness of the world around them, connection with people around them, use of knowledge in practical activities with the aim of transforming the world.

5. Emotional and aesthetic impact, focused on the aesthetic mastery by a person of the world, essence and creativity according to the laws of beauty. The emotional component allows you to enjoy, which significantly changes the lifestyle of a person.

The process of social rehabilitation is a bilateral and counter-process. A healthy society must, for its part, meet the disabled by developing and adapting educational, vocational, labour, cultural, legal, social and other aspects of human existence to the possible inclusion of persons with disabilities in the social environment [1-2]. On the other hand, there should be a desire of people with disabilities to become equal members of society [3].

The article considers the approach to professional training of intellectual persons with disabilities in a holographic manner of a competent healthy specialist in order to increase the motivation and desire of intellectual persons with disabilities to become equal members of society.

\section{Human Holographic Consciousness}

A person, like a hologram, contains everything that is in a multiple updating system from finite shifting professions. The hologram is the basis of a person's life and can become the basis of professional training for intellectual disabled people.

The hologram itself is a three-dimensional photograph taken using a laser. To produce a hologram, first of all, the object to be photographed must be illuminated by laser light. Then the second laser beam, folding with reflected light from the object, gives an interference pattern that can be fixed on the film. The finished shot looks like a pointless alternation of light and dark lines. If you illuminate the shot with another laser beam, a three-dimensional image of the original object appears. The three-dimensional property is inherent in the hologram. If a hologram with an image of an object is cut in half and illuminated with a laser, each half will contain an entire image of the same object. If you continue to cut the hologram into smaller pieces, on each of them we will again find the image of the object as a whole. Unlike conventional photography, each section of the hologram contains information about the entire object, but with a proportionally corresponding decrease in clarity.

The principle of the hologram "everything in each part" allows a fundamentally new approach to the issue of organization and order. The hologram shows that some things 
cannot be investigated by an analytical method: to dissect an object and study its constituent parts. If we dissect anything on the hologram, then we will not get the parts of which it consists, but we will get the same, but with less accuracy.

Our ability to quickly find the right information from the vast volume of our memory is provided by a brain that works on the principle of a hologram. Indeed, one of the most amazing properties of human thinking is that each piece of information is instantly and mutually associated with the other - another property inherent in the hologram.

The location of memory is not the only neurophysiological mystery that has become more solvable in the light of the holographic model of Pribram's brain [3]. The other is how the brain is able to translate such an avalanche of frequencies that it perceives by various sensory organs (frequencies of light, sound frequencies, and so on) into our specific idea of the world. Frequency coding and decoding is exactly what the hologram handles best. Just as the hologram serves as a kind of lens, a transmitting device capable of turning a apparently meaningless frequency interfere into a coherent image, so the brain, according to Pribram, contains such a lens and uses the principles of holography to convert frequencies from the senses into the inner world of our perceptions.

Pribram's idea that our brains construct solid reality by relying on input frequencies has also received experimental confirmation. It has been found that any of our sensory organs has a large frequency range of susceptibility. In particular, the researchers found that our visual organs are susceptible to sound frequencies, that our sense of smell depends somewhat on what is now called osmotic frequencies, and that even our body cells are sensitive to a wide frequency range. This is the work of the holographic part of our consciousness, which converts separate chaotic frequencies into continuous perception.

The holographic model also shows that consciousness is not a function of the brain, but, on the contrary, the neural networks of the brain are controlled by consciousness, which, therefore, does not die if the brain dies. In holographic reality, thought is as real as consciousness.

A single universe has projections in the form of a discretely updated frequency wave-like structure - a hologram, the main content of which is consciousness, which is its copy. Discrete update of the projection by consciousness means the appearance of durations, which qualifies as time.

A single universe enters time by means of its own projection in the form of a frequency wave-like structure - a hologram, forming its own time and at the same time - the general of the entire structure of being due to its holographic essence (the whole and part coincide). The active living in the holographic projection of the Single, copying fragments of the passive from the Single, are combined with them individually and as a whole, and through themselves form the current time, space and things in motion.

The conversion of wave matter (packets of information) from the holographic projection of the Single in any living creature, including in man, into his environment, is carried out by him no longer in the form of images, but in the form of material objects of different "density" and various forms using the corresponding matrices (shaping abilities), creating both the living being itself and its environment within the framework of common time, which is the consequence of the action of a single consciousness in a combined set of living beings.

The formation of objects of various forms in accordance with the mass-forming abilities (matrices) of a living creature is carried out according to the type of work of a 3D printer.

The holographic image of living beings allows them to be separate, living each time their own life, having their own time, their own being, and at the same time in their totality to be a single being that is eternal both in its singularity and in each of its own.

As for the genome, it is based on a program copied by consciousness in a projection from the Unified to the molecular chains of the genome in accordance with its existing shaping abilities for each particular case.

Information is an activity property. Without material objects-carriers, including consciousness itself, information does not exist. For each person, discrete information is automatically (with the help of a single consciousness) converted into things, images, meanings, feelings, which are also quite material components of each individual consciousness, entailing subsequent actions of the carrier of consciousness.

Living beings are involved in the information process. With the help of information processing centers, they are able to immerse themselves in a liveable environment. All human achievements are connected in the form of acquired experience in this field, existing knowledge and skills with a certain target focus.

In the form of light and sound, a person can approach the harmony of his own wave basis of the ideal, which is actually the holographic basis of a person.

The time of any living creature, in particular, and man, is an information process during which material objects are recognized by consciousness by scanning the environment by the processing centers at its disposal. The informational (impulse) nature of time formation is asymmetric (onesided), differing from the usual real or energy interactions of objects in that a living being unilaterally scans the surrounding one, sequentially copying the data recognized and grouped by it. This high-frequency complete update of one position of the next, etc., in particular, does not allow you to go back or run forward due to the asymmetry (onesidedness) and completeness of the update of each position. Therefore, it seems to each person that time flows in one direction: from the past to the future through the present. Due to the complete updating of one position by another, that is, irretrievably and infinitely, in the form of an infinitely updated ultra-high-frequency wave-shaped holographic projection in the form of intermittent moments-durations (pulses of information).

For humans, information passes every moment to brain 
neurons from different human sensory organs in the form of sequences of nerve impulses; each of the pulses contains corresponding information. Immediately after the pulse generation, the nerve fiber is in the so-called refractor state, and cannot be reactivated for 1-2 milliseconds, that is, the nerve fiber is able to conduct nerve pulses with a frequency not higher than 500 hertz. The duration of the pulse itself passing through the nerve fiber is a fraction of a millisecond in each moment.

A pause between nerve impulses containing information means that they do not go continuously. However, this pause is below the threshold of perception by her consciousness and therefore does not fall into it. In particular, the moving picture for human consciousness is provided by an interval between the run-through successive frames of about 0.04 seconds, which is said threshold. Its duration, as you can see, is more than an order of magnitude longer than the duration of the pause. Therefore, the resulting discrete sequential moments (in each of them there is an update of the "reality") of a person's own time, which contain in their sequence all the paintings and all the events of life for a person, merge into a continuous, inseparable flow in his mind.

Each person, starting from the moment of birth, selects through sensations from a diverse environment only those information that can be deciphered, or information, in accordance with the achieved level of consciousness development (shaping abilities) [4].

\section{Holographic Image of a Specialist as Functional Ability}

The specialist is presented in the form of an individual structure and organization. The individual behavior that we directly observe can be considered as an organized and integrated structure of a specialist. The stability of a specialist is his relative immutability and constancy in time and changing situations.

Specific features of mental processes of the person, such as individual manifestations of memory, perception, feelings, thinking, abilities, social experience which joins knowledge acquired by the person skills, abilities and habits are formed mainly in the course of training, have social character.

Individual features of a person's mental processes and social experience are connected and combined into a single structure of a specialist. The most adequate principle of a single structure is the holographic approach to the structure of a specialist.

In the structure of the specialist we will highlight the following main areas:

1. The thought sphere.

2. Emotional-sensual sphere.

3. Cognitive sphere.

4. Communicative sphere.

5. Motivational sphere.

6. Scope of activity and activities.

7. The sphere of creativity.

\section{Status-role sphere.}

The holographic representation of a specialist fully reflects the connection of each of its spheres with other spheres. Among all channels of communication with the surrounding world, a visual channel prevails - visual perception of reality, which allows not only to see physically well, but also to see a distant perspective, to see the goal. For a competent specialist, if not clear now, then he will do everything to be clear.

Specialists live today and feel the desire to get the necessary, mutually linking the sensual and communicative spheres. Motivational sphere and sphere of activity and activity rise to the connection of actualization [5]. The sphere of creativity involves the participation of emotional, cognitive, communicative and mental.

The sphere of typical individual thinking is the structure of the semantic space of activity, given in specific forms of Unity, the integrity of the higher regulatory states of the central nervous system that ensure this activity. Accordingly, the content of a particular individual thinking is the meanings of systemic activity, updated in the current activity of a particular individual and in the specified forms of the integrity of his Central Tax Commission. There is a correspondence between the meanings of systemic activity and the contents of individual thinking. This correspondence, however, is mediated by the specific social and occupational factors that determine this activity. This correspondence determines the meaning of thinking through the holographic dimension of psychic energy [6].

The status role sphere of the specialist is essentially the result of the interaction of the social and production structure of society with the individual [7]. For the stable existence of society, it is necessary that all its status cells be filled with living people. To have workers and farmers, soldiers and guards, pilots and submariners, teachers and doctors, bankers, trolley bus drivers and other specialists. Dreaming of becoming someone from childhood and becoming him, we move as if independently along the chosen path, but conditionally fulfill a social order. Our individuality always fits into a prepared social cell.

The holographic approach to the structure of the specialist fully reflects the connections of his various fields [8].

\section{Holographic Rehabilitation of Intellectual Disability Consciousness}

Magnetic resonance imaging (MRI) is today used not only for diagnosis, but also for mapping the functional state of neural networks, allowing you to literally see the brain's work in real time [9-13].

The ability to directly observe the dynamics of cognitive (cognitive) brain activity appeared only with the introduction of functional magnetic resonance imaging technology into research practice.

A little more than two decades ago, employees of the American research organization AT \& T Bell Laboratories 
described the principle of visualizing the activity of brain zones in real time using magnetic resonance imaging (MRI). A dynamic study of active zones of brain structures at the time of their activity was first tested on a person two years after the first publication.

MRI (magnetic resonance imaging) is a diagnostic procedure based on the effect of nuclear magnetic resonance. Modern MRI techniques allow not only to visualize organs with high quality, but also to investigate their function. Due to the absence of ionizing radiation, this method can be used without restrictions and repeated studies are repeatedly carried out.

It follows that the relative magnitude of the MRI signal can serve as a measure of the activity of brain zones. Moreover, the results obtained under the control of electroencephalography on the visual cortex of the primate open brain suggest that the MRI signal is a linear response to the electrical activity generated by the active neural ensemble.

Functional MRI focused on BOLD effect detection is today the optimal tool for mapping neuronal activity, more precisely, the functional state of neural networks - the basis for visualizing our holographic images in real time.

Closely related to fMRI technology is the neurobiological technology "of the brain-computer interface. We are talking about the possibility with the help of an electroencephalogram to obtain a display of a stable hologram of the bioelectric activity of the brain, tying it to the function of brain structures and the formation of new stable neural ensembles in them. The electroencephalogram is a source of information on intracerebral events. At the same time, fMRI makes it possible to visualize the real temporal and spatial dynamics of the brain. If you briefly describe the most general route of activation of brain structures during internal speech, then after the start, the broad cortical fields of the brain are first involved in the work, and the cognitive route in the cerebellum ends.

Psychology is one of the most promising areas of use of neuroimaging technology by FMRT, because this scientific field is practically devoid of ideas about the localization (in the anatomical sense) of cognitive functions.

In one of the works of American researchers, an attempt was made to answer the question of the localization of brain structures designed to classify such cognitive categories as equality and efficiency. It turned out that the emotional assessment of the "effectiveness," "justice" and "general benefit" of the decision is carried out by three different brain structures. The brain department, called the "shell" (lat. Putamen), is responsible for efficiency, the bark of the "islet" (lat. Insula) protects the interests of justice, a cumulative measure of efficiency and inequality, that is, utility, assesses the septal organ (lat. Septum), while other areas of the brain are involved in the cognitive process.

The development of cognitive science, a direction of neurosciences that studies the basic mechanisms of the brain's work: "mental strategies," their localization, dynamics, ways of using and improving in everyday life, also opens up great prospects. The so-called "interactive stimulation" makes it possible to organize educational (therapeutic) feedback directly through the "interested" brain structure (Figure 1). By visualizing the cingulate gyrus or hippocampus, you get a chance of "direct conversation" with the brain.

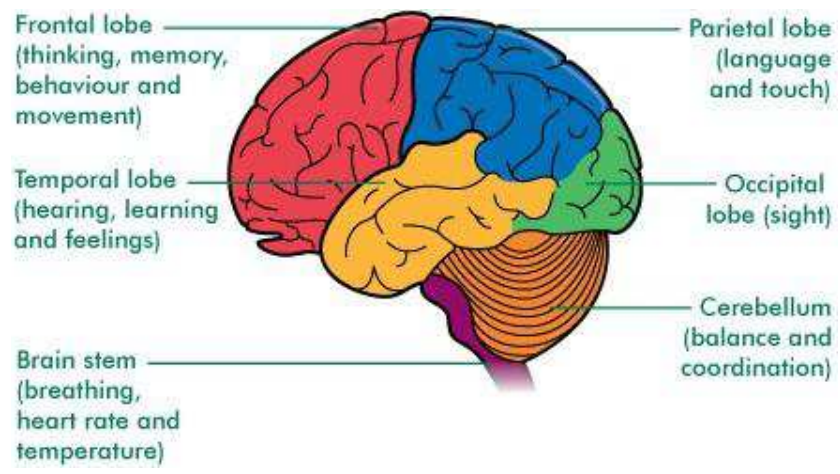

Figure 1. Brain functions.

Knowing how the brain is activated, it is possible to restore the holographic image of a person through a resonant energy trace. Using nanoresonators, the brain activity of a human holographic image can be transmitted through the mental neurointerface of this activity to another person [14-16].

Then it becomes possible to exchange the thoughts of people with similar semantic memory, based on the resonance of equivalent brain activities of thinking. Semantic memories are considered similar if they correspond to the principle of gold section according to professional thesaurus, according to spherodynamics. Spherodynamics is a scientific discipline whose goal is to master the method of volume thinking. The simplest logical and mathematical constructions make it possible to clearly assess the principle of Unity of the World, perfectly known even in ancient times, but practically ignored by most modern scientists.

Formula: $\mathrm{F}=\mathrm{A}^{(2 / 3) 6}$ is a sensor code that exhibits the seven-dimensional structure of the Human sensory space, where $A=240.2402$ is the universal World Constant, $F=1$, (6180..) is the "golden proportion," the famous number of ancient Greek mathematician, philosopher, architect Fidius. At the same time, seven subspaces are resonantly interconnected, and the excitation of one of them, for example, by proper sound extraction, automatically excites the rest. And this Unity with the whole World makes it possible to carry out holographic rehabilitation of the consciousness of an intellectual disabled person by correcting semantic memory in a holographic way of a specialist in the process of comprehensive rehabilitation.

\subsection{Correction of Semantic Memory by Holographic Image of Specialist}

Semantic memory is a holographic image of words, concepts, rules and abstract ideas. Semantic memory has a large number of options for remembering and methods for establishing connections between concepts. Semantic memory is a very flexible, reconstructive, associative, and 
multimodal system. This is crucial in investigating questions regarding cognitive and neural dependencies in supporting cognition more broadly. The holographic image creates a high degree of imagination and specificity energetically with the help of sensory organs. Semantic wealth is a reflection of how many definitions and situations a word or concept is associated with.

Significant advances have appeared in experimental methods about the role of the hippocampus in semantic memory. Hippocampus makes a solid contribution to maintaining semantic memory. Research has deepened the understanding of how words, concepts and meanings, as well as episodes and events, are created and preserved in memory and provide a new understanding of our typical human abilities. Semantic memory in its entirety rests on the hippocampus, but still goes beyond it. In fact, there is significant neuroanatomical overlap (duplication) in the semantic network and the network of the passive mode of operation of the brain. The role of the hippocampus in tracking and determining the relationship between meanings in semantic memory in a holographic manner similar to how the hippocampus tracks and determines the location in the surrounding space. The hippocampus promotes the tracking and construction of semantic associations between words. Scientists claim that the hippocampus plays a role in the prognostic processing of the language. HFB hippocampal power patterns were compared to each other based on semantic similarities calculated using LSA. The results showed that the pre-activation patterns of HFB activity were similar for images that were closer in semantic value to each other [14].

Neurophysiologist, Stanford University professor Karl Pribram, based on holographic theory, explained many other phenomenal brain-related phenomena. Memory, as one of the central functions of the brain, has a distributed rather than localized character, and each part of the brain can contain an integer just as a piece of holographic film contains information on which an entire image is created. The holographic theory explains how the brain manages to store a huge amount of information in a small space. Each biological structure, starting from the cell level, is the source of a wide range of fields. All vibrations or vibrations of internal organs are coherent. It is coherent radiation that creates a holographic image. In biological organism coherent fields form dynamic space-time interference structure-hologram on the basis of reference radiation. The radiation of each point of the object is as a reference relative to all other points.

Only the holographic structure of the brain allows us to explain how information is stored in human memory during clinical death, when the physical body (and the brain in particular) does not function, the potential difference of brain cells is zero. Information, if stored in brain cells, must be erased. And this does not happen, and a person after intensive care still instantly recognizes his relatives and friends, friends and just acquaintances. The holographic model explains the fact of instant recognition. A person, seeing a familiar face, immediately recognizes him. The wave principle of holography allows you to almost instantly extract from the vault the information that is encoded using the wave process.

Russian scientist, academician P. P. Garyaev, creator of a new science - wave genetics - in his book "Wave Genome" notes: human memory has a distinctly pronounced and wellstudied holographic nature.

Academician Treasurer writes: "Today, a paradigm begins to emerge that proclaims that our brain is a hologram, and what we feel and see is a holographic virtual process.

The formation of a multicellular organism is associated with an evolutionary pattern unknown to us, in which each specialized cell, combining into a multicellular structure with other specialized cells, must find the corresponding interaction of holographic fields and the combination of holographic space and time inherent in each cell. The organism is an innumerable combination of various selfdeveloping evolving holographic spaces, fields, and formations. It becomes clear why our brains can hold up to 10 billion bits of different signals in memory. Apparently, the amount of this knowledge is even greater, and the cells of sensory systems, which would seem to have only touch, sense of smell, hearing and vision, respond to other factors with holographic signs.

Berkeley neurophysiologists Russell and Karen Devalua made the crucial discovery. They transformed black and white cells into simple wave forms by the Fourier method. Devalua then conducted experiments to find out how brain cells in the visual part of the cerebral cortex respond to these new wave forms. The result shocked the scientific world: brain cells did not respond to the original images (black and white cells), but to the wave forms of these images. The discovery of Devalua was subsequently confirmed in many laboratories around the world.

Other brain tissue, if necessary, takes part in the electrical interaction between brain nerve cells, or neurons. Neurons have tree branches, and when the electrical signal reaches the end of one such branch, it propagates further in the form of waves, exactly as we observe on the surface of water. Since neurons are closely adjacent to each other, divergent electrical waves are constantly superimposed on each other. Neural holograms are created that are multiple and subtle in nature. Waves can create an endless kaleidoscopic series of interference paintings in which the adaptability of the brain is rooted on the principle of holography. The holographic principle invariably appears in the wave nature of the interaction of brain nerve cells.

The experiments of academician N. P. Bekhtereva showed that brain activity is performed in accordance with quantum laws. They strongly confirmed that the human brain is an organ that generates wave structures that are adequate to forms of the outside world. Based on these studies, physiologist A. N. Lebedev put forward "an assumption about recording perceived information and storing it in memory in the form of stable holographic patterns formed by different phases of coherent non-extinguishing waves of neural activity that appear in different places in the brain. The waves themselves are a combination of different frequency 
oscillations, wherein waves of the same frequency can differ in phases and amplitudes. " The packet of waves of the same frequency with different phases, scientists began to consider the simplest unit of memory.

The source of the holographic recording is wave processes and pulses arising during the operation of nerve cells, while information is encoded on a plurality of neurons interacting with each other. The holographic model perfectly describes the properties of information distribution in brain neural networks. Currently, there is no method that shows the distribution of information at any point in the information store with such clarity and certainty as the mathematical apparatus of holography does.

According to Professor Bergson's hypothesis: "The nervous system and, above all, the brain" extinguishes "most of the sensory stimuli at the entrance of individual consciousness. Moreover, only information that requires close attention and response is selected from them.

The holographic principle also helps explain why people can determine the source of sound without turning their heads. A hologram is a virtual image that has emerged where it does not exist. Our internal speech senses indicate the presence of this virtual image.

Functional magnetic resonance imaging technologies are a powerful tool that allows you to achieve high-quality fixation of a hologram of human brain activity. The introduction of fMRI technologies in various areas of human activity - neuro marketing, professional casting, assessment of the effectiveness of educational programs, will help to professionally correct the semantic memory of an intellectual disabled person in a holographic way of a specialist using mental neurointerfaces and form his holographic professional projection in the semantic memory of a disabled person in the process of comprehensive rehabilitation.

When the consciousness of a rehabilitated intellectual disabled person becomes a holographic professional projection of a specialist, then he is ready for production joint activities through neurointerfaces [15-17].

\subsection{Professional Interaction Through Neurointerfaces}

Mental neurointerfaces are needed for professional interaction in real time, using multi-channel wave nanoresonators, based on the fixation and transmission of a magnetic resonance holographic image. Modern technologies allow us to begin to implement the technology of fixing and transmitting a magnetic resonance holographic image.

Interaction with magnetic resonance holographic images through mental neurointerfaces is ensured in several modes: communication request, communication readiness, holographic image transmission mode, confirmation of mental communication, mental communication testing mode.

A communication request is made to check whether the communication is ready. The mode is activated by a specialist before starting mental communication.

The mode of readiness for communication is introduced to confirm the consent of a rehabilitated intellectual disabled person to mental communication.
The holographic image transmission mode of a specialist is activated after receiving a signal from the recipient of readiness for mental communication. In this mode, the magnetic resonance hologram of the inductor is transmitted to the recipient.

The mental communication confirmation mode is activated after activation of brain activity of the recipient by magnetic resonance hologram of inductor. In this mode, the activation of the holographic image in a rehabilitated intellectual disabled person is confirmed.

The mental communication test mode is entered to configure it. It is turned on to test the activation of the holographic image in the recipient. The test is performed using tests.

After adjustment of mental communication the interlocutors activate interaction with internal speech on the basis of professional information of semantic memory [17].

The technology of communication with holographic images using mental neurointerfaces with two-way communication using multichannel wave nanoresonators allows for professional interaction through neurointerfaces for practical purposes.

The resonant technology of mental communication of people with neurointerfaces ensures the effectiveness of interaction and can be used in various spheres of life. Mental neurointerfaces will have many areas of use and a huge distribution among specialists of various professions and even among the population for industrial interaction. When the mental neurointerface is revealed opportunities, learning and interaction, they will be used by most intellectual disabled people, while all spheres of society will undergo changes. The whole society is morally and technologically prepared for change.

\section{Conclusion}

Global socio-economic and environmental challenges are increasingly facing the international community. In many regions of the Earth, the quality of life is deteriorating, morbidity is increasing, life expectancy is decreasing, and the number of people with disabilities is increasing. There is a need to consider in a new way the key issues of mass recovery of the able-bodied population and people with disabilities.

The analysis of foreign and domestic experience shows the great importance of the rehabilitation process, both for the disabled themselves and for society as a whole. Professional rehabilitation is one of the ways to involve people with disabilities in the active life of society, an effective way to change the position of society towards people with disabilities. In this regard, measures to improve existing and create new effective technologies, rehabilitation and prevention methods that increase the reserve capabilities and abilities of an intellectual disabled person are of particular importance.

The approach to professional training of intellectual disabled persons in the holographic manner of a competent 
healthy specialist, in the process of comprehensive rehabilitation, will increase their motivation and desire to become equal members of society, will help to become professional members of society, thereby reducing the economic burden on society in terms of their content.

\section{References}

[1] Piotr Jankowski, Maciej Niewada, Andrzej Bochenek. Optimal Model of Comprehensive Rehabilitation and Secondary Prevention. September 2013, Kardiologia Polska 71 (9): 9951003.

[2] Lyn Watson, Simon Balster, Sarah A Warby, Jackie Sadi. A comprehensive rehabilitation program for posterior instability of the shoulder. April 2017. Journal of Hand Therapy 30 (2): 182-192.

[3] Carl Pribram. Brain languages. Experimental paradoxes and principles of neuropsychology, Progress, 1975.

[4] Yuri Nizovtsev. A man like a hologram. 2017. https://proza.ru/2017/05/06/1625 Publication Certificate No. 217050601625 .

[5] Maslow A. Motivation and personality. - St. Petersburg: Peter, 2019. - 400 pages.

[6] Zubko A. V. Consciousness as a holographic dimension of mental energy. Ethics and Science of the Future 2014.

[7] Bryndin E. G. Psychological and Social Aspects Formations of Thinking, Consciousness and Behavior. SM Physical Medicine \& Rehabilitation. Volume 2 Issue 1 2018. P. 1-5.

[8] Yontef G. In search of a mirror of living self. Understanding the dialog process. - M.: Ed. MGI, 2019. - 403 pages.
[9] Ilya S. Bakulin, Alexandra Poydasheva. Transcranial magnetic stimulation in cognitive neuroscience: Methodological basis and safety. 2020. DOI: 10.47010/20.3.2.

[10] Lanfang Liu, Xin Yan. Identifying a supramodal language network in human brain with individual fingerprint. NeuroImage, 220 (10). 2020.

[11] Nima Asadi, Yin Wang. A heuristic information cluster search approach for precise functional brain mapping. Human Brain Mapping, 41 (1). 2020.

[12] Xiaoxiao Wang, Xiao Liang. Decoding and mapping task states of the human brain via deep learning. Human Brain Mapping, 41 (6). 2020.

[13] Takumi Mitsuhash, Masaki Sonoda, Jeong-Won Jeong. Fourdimensional tractography animates propagations of neural activation via distinct interhemispheric pathways. 2021. DOI: 10.1016/j.clinph.2020.11.030.

[14] Duff MC, Covington NV, Hilverman C, Cohen NJ. Semantic Memory and the Hippocampus: Revisiting, Reaffirming, and Extending the Reach of Their Critical Relationship. Front Hum Neurosci. 2020; 13: 471. Published 2020 Jan 24. doi: 10.3389/fnhum.2019.00471.

[15] Evgeny Bryndin. Communication of Internal Speech with Communicative Associative Robot via Spectral Neurointerface. Electrical Science \& Engineering. Vol. 3, Issue 1, 2021. pp. 16-22.

[16] Evgeny Bryndin. Mental Communication of Internal Speech with Communicative Associative Robot via Spectral Neurointerface. American Journal of Applied Psychology. Volume 10, Issue 3, 2021. pp. 55-64.

[17] Evgeny Bryndin. Resonance Technology of Communication of Specialists via Mental Neurointerfaces. International Journal of Neurologic Physical Therapy. Vol. 7, Issue 1, 2021. pp. 7-13. 